\title{
The crystal structure of dichlorido(1,3-bis(2,6- diisopropyl-phenyl)-1H-3 $\lambda^{4}$-imidazol-2-yl)(3- phenyl-pyridine- $\mathrm{KN}$ ) palladium(IV), $\mathrm{C}_{38} \mathrm{H}_{45} \mathrm{~N}_{3} \mathrm{Cl}_{2} \mathrm{Pd}$
}

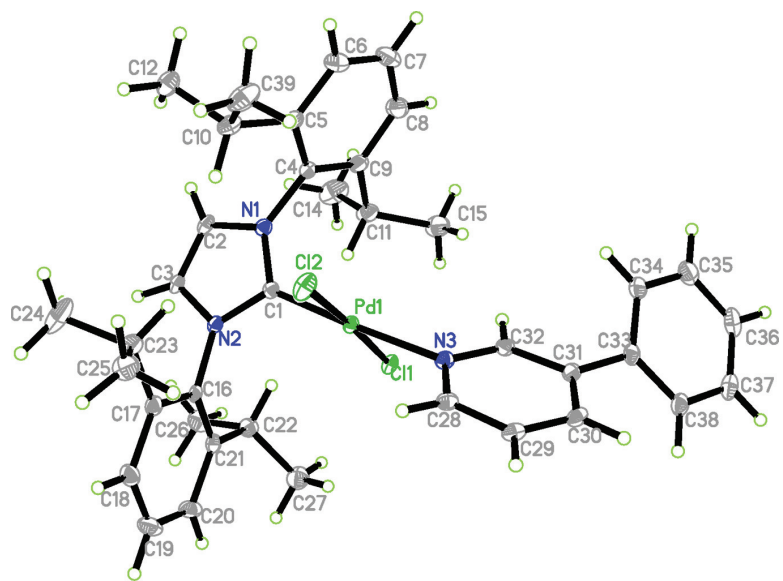

https://doi.org/10.1515/ncrs-2019-0050

Received January 16, 2019; accepted March 9, 2019; available online April 27, 2019

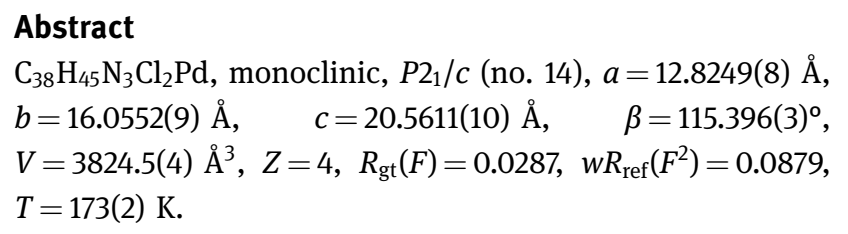

CCDC no.: 1902125

The molecular structure is shown in the figure. Table 1 contains crystallographic data and Table 2 contains the list of the atoms including atomic coordinates and displacement parameters.

\section{Source of material}

According to Shao and co-workers' previously reported method, the title product was obtained in good yield [4]. Crystals suitable for X-ray diffraction were grown in a mixture of ethyl acetate and petroleum ether.

\footnotetext{
*Corresponding author: Ping Lin, College of Chemistry and Materials Engineering, Wenzhou University, Chashan University Town, Wenzhou Zhejiang Province, 325035, China, e-mail: 110534015@qq.com

Zhen Bao: College of Chemistry and Materials Engineering, Wenzhou University, Chashan University Town, Wenzhou Zhejiang Province, 325035, P.R. China
}

๑ Open Access. ( 2019 Zhen Bao et al., published by De Gruyter. (c) BY License.
Table 1: Data collection and handling.

\begin{tabular}{ll}
\hline Crystal: & Colourless block \\
Size: & $0.30 \times 0.25 \times 0.20 \mathrm{~mm}$ \\
Wavelength: & Mo $K \alpha$ radiation $(0.71073 \AA)$ \\
$\mu:$ & $0.65 \mathrm{~mm}^{-1}$ \\
Diffractometer, scan mode: & Bruker APEX II, $\varphi$ and $\omega$ \\
$\theta_{\text {max }}$, completeness: & $26.0^{\circ},>99 \%$ \\
$N(h k l)_{\text {measured }}, N(h k l)_{\text {unique }}, R_{\text {int }}:$ & $31228,7483,0.038$ \\
Criterion for $I_{\text {obs }}, N(h k l)_{\text {gt }}:$ & $I_{\text {obs }}>2 \sigma\left(I_{\text {obs }}\right), 6525$ \\
$N(\text { param })_{\text {refined }}:$ & 405 \\
Programs: & Bruker [1], SHELX [2], Olex2 [3] \\
\hline
\end{tabular}

\section{Experimental details}

All hydrogen atoms attached to $\mathrm{C}$ atoms were introduced using the HFIX command in the SHELXL-2016/6 program [2, 3, 5]. The $\mathrm{C}-\mathrm{H}$ distances in $\mathrm{CH}_{3}$ were restrained to $0.98 \AA$ with $U_{\text {iso }}$ values to be $1.5 U_{\text {eq }}(\mathrm{C})$. Vinylic and aromatic $\mathrm{C}-\mathrm{H}$ distances were restrained to $0.95 \AA$ with $U_{\text {iso }}$ values to be $1.2 U_{\text {eq }}(\mathrm{C})$. The $\mathrm{C}-\mathrm{H}$ distances in $\mathrm{CH}$ in the isopropyl groups were restrained to $1.00 \AA$ A with $U_{\text {iso }}$ values to be $1.2 U_{\text {eq }}(\mathrm{C})[1,6]$.

\section{Comment}

Since the preliminary report on the monodentate $N$-heterocyclic carbene-palladium complexes using pyridines as the throw-away ligands was found $[7,8]$, various such analogues using pyridines as the ancillary ligands have been reported to date [9-13]. According to $\mathrm{Lu}$ and some others' work, subtle change on the anicillary ligands will also have significant effect on the catalytic activities of such complexes [14-22]. Herein, we report the crystal structure of the title compound using 3-phenyl-pyridine as the ancillary ligand.

In the crystal structure, the same coordinate mode of the Pd center was obtained compared to those previously reported analogues. The structural parameters around the $\mathrm{Pd}$ center are also very similar with those reported ones [23]. For instance, the $\mathrm{Pd}$ center also showed slightly distorted square-planar geometry with four ligands. The bond lengths around the $\mathrm{Pd}$ centers are: $\operatorname{Pd}(1)-\mathrm{C}(1)=1.971(2) \AA$; $\operatorname{Pd}(1)-\mathrm{N}(3)=2.0985(19) \AA \AA \operatorname{Pd}(1)$ $\mathrm{Cl}(1)=2.3011(6) \AA$; $\quad \operatorname{Pd}(1)-\mathrm{Cl}(2)=2.3047(6) \AA$. $\quad$ The bond angles around the Pd center are: $\mathrm{C}(1)-\mathrm{Pd}(1)-\mathrm{N}(3)=176.02(8)^{\circ}$; 
Table 2: Fractional atomic coordinates and isotropic or equivalent isotropic displacement parameters $\left(\AA^{2}\right)$.

\begin{tabular}{|c|c|c|c|c|}
\hline Atom & $x$ & $y$ & $z$ & $U_{\text {iso }} * / U_{\text {eq }}$ \\
\hline d1 & $0.39119(2)$ & $0.34061(2)$ & $0.59835(2)$ & $0.01654(7)$ \\
\hline $\mathrm{Cl} 1$ & $0.39152(5)$ & $0.26321(3)$ & $0.50424(3)$ & $0.02117(13)$ \\
\hline $\mathrm{Cl} 2$ & $0.40178(6)$ & $0.41986(4)$ & $0.69445(3)$ & $0.03110(15)$ \\
\hline N1 & $0.31500(16)$ & $0.19104(12)$ & $0.65283(10)$ & $0.0188(4)$ \\
\hline N2 & $0.49940(16)$ & $0.18828(12)$ & $0.68819(10)$ & $0.0173(4)$ \\
\hline N3 & $0.36909(16)$ & $0.44886(12)$ & $0.53663(11)$ & $0.0201(4)$ \\
\hline $\mathrm{C} 1$ & $0.40344(19)$ & $0.23533(14)$ & $0.65041(11)$ & $0.0172(4)$ \\
\hline$C 2$ & $0.3558(2)$ & $0.11707(14)$ & $0.69078(12)$ & $0.0216(5)$ \\
\hline $\mathrm{H} 2$ & 0.310731 & 0.075592 & 0.699752 & $0.026^{\star}$ \\
\hline C3 & $0.4698(2)$ & $0.11529(14)$ & $0.71217(12)$ & $0.0209(5)$ \\
\hline H3 & 0.521048 & 0.072149 & 0.738808 & $0.025^{*}$ \\
\hline C4 & $0.1947(2)$ & $0.21511(15)$ & $0.62300(13)$ & $0.0232(5)$ \\
\hline C5 & $0.1545(2)$ & $0.25613(16)$ & $0.66792(14)$ & $0.0278(5)$ \\
\hline C6 & $0.0366(3)$ & $0.2748(2)$ & $0.63795(17)$ & $0.0442(8)$ \\
\hline H6 & 0.006001 & 0.303640 & 0.666224 & $0.053^{*}$ \\
\hline $\mathrm{C7}$ & $-0.0360(3)$ & $0.2525(3)$ & $0.56859(18)$ & $0.0574(10)$ \\
\hline $\mathrm{H} 7$ & -0.115808 & 0.265709 & 0.549631 & $0.069^{\star}$ \\
\hline $\mathrm{C} 8$ & $0.0070(3)$ & $0.2109(2)$ & $0.52633(16)$ & $0.0506(9)$ \\
\hline $\mathrm{H} 8$ & -0.044295 & 0.195749 & 0.478566 & $0.061 *$ \\
\hline C9 & $0.1228(2)$ & $0.19079(19)$ & $0.55185(14)$ & $0.0319(6)$ \\
\hline C10 & $0.1688(2)$ & $0.14152(18)$ & $0.50651(14)$ & $0.0337(6)$ \\
\hline $\mathrm{H} 10$ & 0.253916 & 0.151447 & 0.526965 & $0.040^{\star}$ \\
\hline C11 & $0.1165(3)$ & $0.1704(2)$ & $0.42766(16)$ & $0.0452(8)$ \\
\hline $\mathrm{H} 11 \mathrm{~A}$ & 0.036521 & 0.150996 & 0.403151 & $0.068^{*}$ \\
\hline $\mathrm{H} 11 \mathrm{~B}$ & 0.161529 & 0.147363 & 0.403601 & 0.068 * \\
\hline $\mathrm{H} 11 \mathrm{C}$ & 0.118132 & 0.231406 & 0.425954 & $0.068^{*}$ \\
\hline C12 & $0.1505(3)$ & $0.0482(2)$ & $0.51054(19)$ & $0.0527(9)$ \\
\hline $\mathrm{H} 12 \mathrm{~A}$ & 0.184787 & 0.030492 & 0.561025 & $0.079^{\star}$ \\
\hline H12B & 0.187422 & 0.018126 & 0.484512 & $0.079 *$ \\
\hline $\mathrm{H} 12 \mathrm{C}$ & 0.067660 & 0.036003 & 0.488658 & $0.079^{\star}$ \\
\hline C13 & $0.2323(2)$ & $0.27557(16)$ & $0.74613(14)$ & $0.0287(6)$ \\
\hline H13 & 0.313824 & 0.276109 & 0.752016 & $0.034^{*}$ \\
\hline C14 & $0.2071(3)$ & $0.36034(18)$ & $0.77012(18)$ & $0.0451(8)$ \\
\hline $\mathrm{H} 14 \mathrm{~A}$ & 0.132460 & 0.358365 & 0.772498 & $0.068^{\star}$ \\
\hline H14B & 0.204810 & 0.403049 & 0.735474 & $0.068^{\star}$ \\
\hline $\mathrm{H} 14 \mathrm{C}$ & 0.267952 & 0.373949 & 0.817755 & 0.068 * \\
\hline C15 & $0.2219(3)$ & $0.20750(17)$ & $0.79504(14)$ & $0.0325(6)$ \\
\hline $\mathrm{H} 15 \mathrm{~A}$ & 0.143020 & 0.206604 & 0.791059 & 0.049 * \\
\hline H15B & 0.276172 & 0.218917 & 0.845021 & $0.049^{\star}$ \\
\hline $\mathrm{H} 15 \mathrm{C}$ & 0.240017 & 0.153344 & 0.780383 & $0.049^{*}$ \\
\hline C16 & $0.61764(19)$ & $0.21177(14)$ & $0.70633(12)$ & $0.0189(5)$ \\
\hline C17 & $0.6763(2)$ & $0.25878(15)$ & $0.76902(13)$ & $0.0232(5)$ \\
\hline C18 & $0.7925(2)$ & $0.27553(16)$ & $0.78841(14)$ & $0.0297(6)$ \\
\hline H18 & 0.835743 & 0.305958 & 0.831230 & $0.036^{\star}$ \\
\hline C19 & $0.8459(2)$ & $0.24860(17)$ & $0.74637(16)$ & $0.0348(6)$ \\
\hline H19 & 0.924635 & 0.262067 & 0.759930 & $0.042^{\star}$ \\
\hline $\mathrm{C} 20$ & $0.7865(2)$ & $0.20263(16)$ & $0.68527(15)$ & $0.0300(6)$ \\
\hline $\mathrm{H} 2 \mathrm{O}$ & 0.825175 & 0.184816 & 0.657340 & $0.036^{*}$ \\
\hline $\mathrm{C} 21$ & $0.6699(2)$ & $0.18117(15)$ & $0.66288(13)$ & $0.0226(5)$ \\
\hline C22 & $0.6085(2)$ & $0.12357(15)$ & $0.59941(14)$ & $0.0266(5)$ \\
\hline $\mathrm{H} 22$ & 0.523448 & 0.129994 & 0.583280 & $0.032^{*}$ \\
\hline C23 & $0.6360(3)$ & $0.14438(19)$ & $0.53585(16)$ & $0.0388(7)$ \\
\hline $\mathrm{H} 23 \mathrm{~A}$ & 0.622865 & 0.203878 & 0.524701 & $0.058^{*}$ \\
\hline H23B & 0.585840 & 0.111707 & 0.493755 & $0.058^{*}$ \\
\hline $\mathrm{H} 23 \mathrm{C}$ & 0.716875 & 0.130873 & 0.548364 & $0.058^{\star}$ \\
\hline
\end{tabular}

Table 2 (continued)

\begin{tabular}{lrrrr}
\hline Atom & $\boldsymbol{x}$ & $\boldsymbol{y}$ & $\boldsymbol{z}$ & $\boldsymbol{U}_{\text {iso }} / \boldsymbol{U}_{\text {eq }}$ \\
\hline C24 & $0.6411(2)$ & $0.03294(16)$ & $0.62337(16)$ & $0.0353(6)$ \\
H24A & 0.725039 & 0.026339 & 0.642721 & $0.053^{*}$ \\
H24B & 0.603454 & -0.004224 & 0.582003 & $0.053^{*}$ \\
H24C & 0.615708 & 0.018855 & 0.660684 & $0.053^{*}$ \\
C25 & $0.6178(2)$ & $0.28740(17)$ & $0.81550(14)$ & $0.0316(6)$ \\
H25 & 0.532804 & 0.290157 & 0.784435 & $0.038^{*}$ \\
C26 & $0.6578(2)$ & $0.37383(18)$ & $0.84868(15)$ & $0.0354(6)$ \\
H26A & 0.737945 & 0.370612 & 0.885115 & $0.053^{*}$ \\
H26B & 0.608466 & 0.392975 & 0.871223 & $0.053^{*}$ \\
H26C & 0.652456 & 0.413120 & 0.810906 & $0.053^{*}$ \\
C27 & $0.6384(4)$ & $0.2243(2)$ & $0.87517(17)$ & $0.0563(10)$ \\
H27A & 0.610173 & 0.169542 & 0.853883 & $0.085^{*}$ \\
H27B & 0.596983 & 0.241935 & 0.903339 & $0.085^{*}$ \\
H27C & 0.721173 & 0.220967 & 0.906676 & $0.085^{*}$ \\
C28 & $0.43413(19)$ & $0.51750(14)$ & $0.56236(13)$ & $0.0207(5)$ \\
H28 & 0.487610 & 0.519367 & 0.611662 & $0.025^{*}$ \\
C29 & $0.4251(2)$ & $0.58555(15)$ & $0.51875(13)$ & $0.0227(5)$ \\
H29 & 0.473259 & 0.632755 & 0.537828 & $0.027^{*}$ \\
C30 & $0.3456(2)$ & $0.58439(14)$ & $0.44738(13)$ & $0.0231(5)$ \\
H30 & 0.339118 & 0.630481 & 0.416911 & $0.028^{*}$ \\
C31 & $0.2749(2)$ & $0.51484(14)$ & $0.42059(13)$ & $0.0212(5)$ \\
C32 & $0.29128(19)$ & $0.44853(14)$ & $0.46747(12)$ & $0.0200(5)$ \\
H32 & 0.244793 & 0.400292 & 0.449540 & $0.024^{*}$ \\
C33 & $0.1863(2)$ & $0.50770(15)$ & $0.34503(13)$ & $0.0240(5)$ \\
C34 & $0.2096(2)$ & $0.53182(15)$ & $0.28702(14)$ & $0.0271(5)$ \\
H34 & 0.281593 & 0.556597 & 0.295669 & $0.032^{*}$ \\
C35 & $0.1272(2)$ & $0.51943(17)$ & $0.21699(14)$ & $0.0341(6)$ \\
H35 & 0.143498 & 0.535501 & 0.177859 & $0.041^{*}$ \\
C36 & $0.0218(2)$ & $0.48409(17)$ & $0.20346(15)$ & $0.0380(7)$ \\
H36 & -0.033402 & 0.475003 & 0.155286 & $0.046^{*}$ \\
C37 & $-0.0032(2)$ & $0.46186(17)$ & $0.26034(16)$ & $0.0366(6)$ \\
H37 & -0.076374 & 0.438827 & 0.251262 & $0.044^{*}$ \\
C38 & $0.0789(2)$ & $0.47333(15)$ & $0.33053(14)$ & $0.0290(6)$ \\
H38 & 0.061599 & 0.457495 & 0.369339 & $0.035^{*}$ \\
\hline & & & &
\end{tabular}

$\mathrm{C}(1)-\mathrm{Pd}(1)-\mathrm{Cl}(1)=88.00(6)^{\circ} ; \quad \mathrm{N}(3)-\mathrm{Pd}(1)-\mathrm{Cl}(1)=89.26(5)^{\circ}$; $\mathrm{C}(1)-\operatorname{Pd}(1)-\mathrm{Cl}(2)=92.64(6)^{\circ} ; \quad \mathrm{N}(3)-\mathrm{Pd}(1)-\mathrm{Cl}(2)=90.26(5)^{\circ}$; $\mathrm{Cl}(1)-\mathrm{Pd}(1)-\mathrm{Cl}(2)=176.73(2)^{\circ}$. In addition, it is worthy of noting here that initial studies on the catalytic activity of the title compound toward the amination reactions between primary and secondary amines with aryl chlorides were examined. It was found that the title compound was a very efficient catalyst for such transformation even at very low catalyst loadings (0.01-0.005 mol\%).

Acknowledgements: We acknowledge the financial support from Wenzhou University for the publication fee.

\section{References}

1. Bruker. SADABS, Version 2.03, SAINT, Version 6.02. Bruker AXS Inc., Madison, WI, USA (2002).

2. Sheldrick, G. M.: Crystal structure refinement with SHELXL. Acta Crystallogr. C71 (2015) 3-8. 
3. Dolomanov, O. V.; Bourhis, L. J.; Gildea, R. J.; Howard, J. A. K.; Puschmann, H.: OLEX2: a complete structure solution, refinement and analysis program. J. Appl. Crystallogr. 42 (2009) 339-341.

4. Zhu, L.; Gao, T.-T.; Shao, L.-X.: Well-defined NHC-Pd(II)-Im (NHC $=N$-heterocyclic carbene; $I \mathrm{~m}=1$-methylimidazole) complexes catalyzed amination of aryl chlorides. Tetrahedron 67 (2011) 5150-5155.

5. Burla, M. C.; Caliandro, R.; Camalli, M.; Carrozzini, B.; Cascarano, G. L.; De Caro, L.; Giacovazzo, C.; Polidori, G.; Spagna, R.: IL MILIONE: a suite of computer programs for crystal structure solution of proteins. J. Appl. Crystallogr. 40 (2007) 609-613.

6. Spek, A. L.: Structure validation in chemical crystallography. Acta Crystallogr. D65 (2009) 148-155.

7. Bertogg, A.; Camponovo, F.; Togni, A.: N-Ferrocenyl-substituted planar-chiral $N$-heterocyclic carbenes and their Pd" complexes. Eur. J. Inorg. Chem. 2005 (2005) 347-356.

8. O’Brien, C. J.; Kantchev, E. A. B.; Valente, C.; Hadei, N.; Chass, G. A.; Lough, A.; Hopkinson, A. C.; Organ, M. G.: Easily prepared air- and moisture-stable Pd-NHC $(\mathrm{NHC}=\mathrm{N}$-heterocyclic carbene) complexes: a reliable, user-friendly, highly active palladium precatalyst for the Suzuki-Miyaura reaction. Chem. Eur. J. 12 (2006) 4743-4748.

9. Sharif, S.; Rucker, R. P.; Chandrasoma, N.; Mitchell, D.; Rodriguez, M. J.; Froese, R. D. J.; Organ, M. G.: Selective monoarylation of primary amines using the Pd-PEPPSI-IPent ${ }^{\mathrm{Cl}}$ precatalyst. Angew. Chem. Int. Ed. 54 (2015) 9507-9511.

10. Krinsky, J. L.; Martinez, A.; Godard, C.; Castillon, S.; Claverarbenes, C.: Modular synthesis of functionalisable alkoxytethered $\mathrm{N}$-heterocyclic carbene ligands and an active catalyst for Buchwald-Hartwig aminations. Adv. Synth. Catal. 356 (2014) 460-474.

11. Zhang, Y.; Cesar, V.; Storch, G.; Lugan, N.; Lavigne, G.: Skeleton decoration of NHCs by amino groups and its sequential booster effect on the palladium-catalyzed Buchwald-Hartwig amination. Angew. Chem. Int. Ed. 53 (2014) 6482-6486.

12. Pompeo, M.; Farmer, J. L.; Froese, R. D. J.; Organ, M. G.: Room-temperature amination of deactivated aniline and aryl halide partners with carbonate base using a Pd-PEPPSIIPent ${ }^{\mathrm{Cl}}$-o-picoline catalyst. Angew. Chem. Int. Ed. 53 (2014) 3223-3226.

13. Fang, W.-W.; Jiang, J.; Xu, Y.; Zhou, J.-F.; Tu, T.: Novel robust benzimidazolylidene palladium complexes: synthesis, structure, and catalytic applications in amination of chloroarenes. Tetrahedron 69 (2013) 673-679.

14. Huang, P.; Wang, Y.-X.; Yu, H.-F.; Lu, J.-M.: $N$-Heterocyclic carbene-palladium(II)-4,5-dihydrooxazole complexes: synthesis and catalytic activity toward amination of aryl chlorides. Organometallics 33 (2014) 1587-1593.

15. Liu, F.; Zhu, Y.-R.; Song, L.-G.; Lu, J.-M.: Synthesis of $\mathrm{N}$-heterocyclic carbene- $\mathrm{PdCl}_{2}$-(iso)quinoline complexes and their application in arylamination at low catalyst loadings. Org. Biomol. Chem. 14 (2016) 2563-2571.

16. Zhao, X.-Y.; Zhou, Q.; Lu, J.-M.: Synthesis and characterization of $N$-heterocyclic carbene-palladium(II) chlorides-1methylindazole and -1-methylpyrazole complexes and their catalytic activity toward C $-\mathrm{N}$ coupling of aryl chlorides. RSC Adv. 6 (2016) 24484-24490.

17. Zhang, Z.-M.; Gao, Y.-J.; Lu, J.-M.: Synthesis of $N$-heterocyclic carbene-Pd(II) complexes and their catalytic activity in the Buchwald-Hartwig amination of aryl chlorides. Tetrahedron 73 (2017) 7308-7314.

18. Liu, F.; Hu, Y.-Y.; Li, D.; Zhou, Q.; Lu, J.-M.: N-Heterocyclic carbene-palladacyclic complexes: synthesis, characterization and their applications in the $\mathrm{C}-\mathrm{N}$ coupling and $\alpha$-arylation of ketones using aryl chlorides. Tetrahedron 74 (2018) 5683-5690.

19. Sun, K.-X.; He, Q.-W.; Xu, B.-B.; Wu, X.-T.; Lu, J.-M.: Synthesis of $\mathrm{N}$-heterocyclic carbene-Pd"-2-methyl-4,5-dihydrooxazole complexes and their application toward highly chemoselective mono-Suzuki-Miyaura coupling of dichlorobenzenes. Asian J. Org. Chem. 7 (2018) 781-787.

20. Wang, Z.-Y.; Ma, Q.-N.; Li, R.-H.; Shao, L.-X.: Palladiumcatalyzed Suzuki-Miyaura coupling of aryl sulfamates with arylboronic acids. Org. Biomol. Chem. 11 (2013) 7899-7906.

21. Chen, M.-T.; Vicic, D. A.; Turner, M. L.; Navarro, O.: ( $N$-Heterocyclic carbene) $\mathrm{PdCl}_{2}$ (TEA) complexes: studies on the effect of the throw-away ligand in catalytic activity. Organometallics 30 (2011) 5052-5056.

22. Chen, M.-T.; Vicic, D. A.; Chain, W. J.; Turner, M. L.; Navarro, O.: Inhibited catalyst activation in $(\mathrm{N}$-heterocyclic carbene) $\mathrm{PdCl}_{2}$ (diethylamine) complexes by intramolecular hydrogen bonding. Organometallics 30 (2011) 6770-6773.

23. Yang, J.: The crystal structure of dichlorido(1,3-dimesityl$1 H-3 \lambda^{4}$-imidazol-2-yl)(morpholine-kN)palladium(IV), $\mathrm{C}_{25} \mathrm{H}_{33} \mathrm{Cl}_{2} \mathrm{~N}_{3}$ OPd. Z. Kristallogr. NCS 233 (2018) 1005-1006. 